TRANSACTIONS OF THE

AMERICAN MATHEMATICAL SOCIETY

Volume 281, Number I, January 1984

\title{
NONLINEAR STABILITY OF ASYMPTOTIC SUCTION ${ }^{1}$
}

\author{
BY \\ MILAN MIKLAVČIČ
}

\begin{abstract}
The semigroup approach to the Navier-Stokes equation in halfspace is used to prove that the stability of the asymptotic suction velocity profile is determined by the eigenvalues of the classical Orr-Sommerfeld equation. The usual obstacle, namely, that the corresponding linear operator contains 0 in the spectrum is removed with the use of weighted spaces.
\end{abstract}

1. Introduction. A flow over a plane $y=0$ in $\mathbf{R}^{3}$ given by

$$
U(x, y, z)=\left(1-e^{-y},-1 / R, 0\right)
$$

is called an asymptotic suction velocity profile [12]. $R>0$ is the Reynolds number. $U$ satisfies the Navier-Stokes equation

$$
\frac{\partial v}{\partial t}+(v \cdot \nabla) v=-\nabla p_{0}+\frac{1}{R} \Delta v, \quad \operatorname{div} v=0
$$

with $p_{0}=0$. In the present paper it is proved that the stability of $U$ for small perturbations which initially decay exponentially in the $y$ direction and are periodic in the $x$ and $z$ directions is governed by the eigenvalues of the classical Orr-Sommerfeld equation $[1,8,12]$. For precise statements see Theorems 4, 5, 9 and 15.

Rigorous nonlinear stability analyses for flows in (essentially) unbounded domains are usually based on energy methods $[3,4,5,13]$, and predict sufficient conditions for global stability [8]. Recently, Galdi and Rionero [6] proved a universal sufficient condition for global stability and it applies, in particular, to the asymptotic suction. However, much importance is attached to (conditional) stability analysis of some special flows over an infinite flate plate $[1,8,12]$. In these cases the most widely accepted stability analyses are based on studies of the eigenvalues of the OrrSommerfeld equation $[1,8,12]$, but no rigorous justification was known.

Let $h_{a}$ denote the function $h_{a}(x, y, z)=e^{-a y}$ for $y \geqslant 0$ and $a \in \mathbf{C}$. Assuming that $\omega>0$ and that

$$
v=\left(1-h_{1}+h_{\omega} u_{1},-1 / R+h_{\omega} u_{2}, h_{\omega} u_{3}\right),
$$

Received by the editors January 3, 1983.

1980 Mathematics Subject Classification. Primary 35Q10; Secondary 76E05, 47A10.

${ }^{1}$ This work was supported in part by Department of Energy grant number DE-AS05-80ER10711.

(C) 1984 American Mathematical Society $0002-9947 / 84 \$ 1.00+\$ .25$ per page 
$p_{0}=h_{\omega} p$ is a solution of the Navier-Stokes equation, we find the following equations for $u=\left(u_{1}, u_{2}, u_{3}\right)$ and $p$ :

$$
\begin{gathered}
\frac{\partial u}{\partial t}-\frac{1}{R} \Delta u+\frac{2 \omega-1}{R} \frac{\partial u}{\partial y}+\left(1-h_{1}\right) \frac{\partial u}{\partial x}+\frac{\omega-\omega^{2}}{R} u \\
+\left(h_{1} u_{2}+\frac{\partial p}{\partial x}, \frac{\partial p}{\partial y}-\omega p, \frac{\partial p}{\partial z}\right)+h_{\omega}\left(u_{1} \frac{\partial u}{\partial x}+u_{2}\left(\frac{\partial u}{\partial y}-\omega u\right)+u_{3} \frac{\partial u}{\partial z}\right)=0, \\
\frac{\partial u_{1}}{\partial x}+\frac{\partial u_{3}}{\partial z}+\frac{\partial u_{2}}{\partial y}-\omega u_{2}=0 .
\end{gathered}
$$

This set of equations (perturbation equations) will be studied in the present paper. As mentioned above, $u$ and $p$ are assumed to be periodic in $x$ and $z$. In $\S 2$ the Fourier components for $u$ and $p$ are analyzed. The full problem is studied in $\S 3$. In $\S 3.5$ the corresponding abstract semilinear parabolic equation is introduced and analyzed. In $\$ 3.6$ it is shown that all solutions of the abstract problem are infinitely differentiable in time in the classical sense. In order to preserve simplicity no attempt has been made to obtain classical spatial smoothness [2]. Therefore, the results presented in $\$ 3.5$ apply to the perturbation equations, provided that the space derivatives are obtained by termwise differentiation of the Fourier series.

I am indebted to M. Ahrens, R. C. DiPrima, G. P. Galdi, D. D. Joseph, S. Rionero, H. Weinberger and P. F. Zweifel for several illuminating discussions.

\section{Fourier components.}

2.1 Preliminaries I. Throughout $\mathcal{H}\left(\mathcal{H}^{j}\right)$ denotes the Hilbert space $L^{2}(0, \infty)$ ( $j$-fold product of $L^{2}(0, \infty)$ ) and $\|\cdot\|$ represents either the norm in $\mathcal{H}$ or $\mathcal{H}^{j}$, depending on the context. The set of all complex valued functions which are absolutely continuous on $[0, a]$ for every $a>0$ is denoted by $Q \mathcal{C}$.

Several operators on $\mathcal{H}$ appear frequently and are defined as follows:

$$
\begin{aligned}
& \mathscr{D}\left(T_{0}\right)=\left\{f \in \mathcal{Q C} \cap \mathcal{H} \mid f^{\prime} \in \mathcal{H}\right\}, \quad T_{0} f=f^{\prime}, \\
& \mathscr{D}\left(T_{1}\right)=\left\{f \in \mathscr{D}\left(T_{0}\right) \mid f(0)=0\right\}, \quad T_{1} f=f^{\prime}, \\
& \mathscr{D}(T)=\left\{f \in \mathscr{D}\left(T_{1}\right) \mid T_{1} f \in \mathscr{D}\left(T_{0}\right)\right\}, \quad T f=-f^{\prime \prime} .
\end{aligned}
$$

For $\operatorname{Re}(z)>0$ define $F(z), G(z) \in \mathscr{B}(\mathcal{H})$ by

$$
(F(z) g)(x)=\int_{0}^{x} e^{z(s-x)} g(s) d s, \quad(G(z) g)(x)=\int_{x}^{\infty} e^{z(x-s)} g(s) d s .
$$

In the obvious manner define operators $T_{1}^{(j)}, T^{(j)}: \mathcal{H}^{j} \rightarrow \mathcal{H}^{j}$ by

$$
T_{1}^{(j)}=T_{1} \oplus \cdots \oplus T_{1} \quad \text { and } \quad T^{(j)}=T \oplus \cdots \oplus T .
$$

Observe that $F(z)=\left(z+T_{1}\right)^{-1}$ and $G(z)=\left(z-T_{0}\right)^{-1}$ for $\operatorname{Re}(z)>0$. The function $h_{a}(x)=e^{-a x}, a \in \mathbf{C}, x \geqslant 0$, will be usually considered as a multiplication operator on $\mathcal{H}$ with its maximal domain.

Lemma 1. Suppose $\boldsymbol{\xi} \in \mathbf{C}, \gamma \in \mathbf{R}, R>0$ and $S=T / R+(2 \gamma-1) T_{1} / R+\xi$. Then:

(a) $z \in \sigma(S)$ iff $R(\operatorname{Im}(z-\xi))^{2} \leqslant(2 \gamma-1)^{2} \operatorname{Re}(z-\xi)$ and $\operatorname{Re}(z) \geqslant \operatorname{Re}(\xi)$.

(b) If $\gamma \geqslant \frac{1}{2}$ then $\sigma_{p}(S)=\varnothing$. 
(c) If $\gamma<\frac{1}{2}$ then $z \in \sigma(S) \backslash \sigma_{p}(S)$ iff $R(\operatorname{Im}(z-\xi))^{2}=(2 \gamma-1)^{2} \operatorname{Re}(z-\xi)$.

(d) If $z \notin \sigma(S)$ and $t=\sqrt{\left(\gamma-\frac{1}{2}\right)^{2}-R(z-\xi)}$ with $\operatorname{Re}(t) \geqslant 0$ then $(S-z)^{-1}=$ $R F\left(t-\gamma+\frac{1}{2}\right) G\left(t+\gamma-\frac{1}{2}\right)$.

(e) If $\gamma=\frac{1}{2}, \phi \in(0, \pi / 2], r>0, a \leqslant \operatorname{Re}(\xi)-(|\operatorname{Im}(\xi)| \cos \phi+r) / \sin \phi$ and $|\arg (z-a)| \in[\phi, \pi]$ then $z \notin \sigma(S)$ and

$$
\begin{aligned}
\left\|(S-z)^{-1}\right\| & \leqslant \frac{1}{r}, \\
\left\|(S-z)^{-1}\right\| & \leqslant \frac{1}{|z-a|} \frac{|\operatorname{Im}(\xi)|+r}{r \sin \phi}, \\
\left\|T(S-z)^{-1}\right\| & \leqslant \frac{R}{\sin \phi} .
\end{aligned}
$$

Proof. Conclusions (a) through (d) are obvious. Using the spectral resolution of $T$ one can easily obtain

$$
\left\|(T-z)^{-1}\right\| \leqslant \begin{cases}1 /|\operatorname{Im}(z)| & \text { if } \operatorname{Re}(z) \geqslant 0 \\ 1 /|z| & \text { if } \operatorname{Re}(z)<0\end{cases}
$$

and

$$
\left\|T(T-z)^{-1}\right\| \leqslant \begin{cases}|z| /|\operatorname{Im}(z)| & \text { if } \operatorname{Re}(z) \geqslant 0 \\ 1 & \text { if } \operatorname{Re}(z)<0\end{cases}
$$

These bounds imply (e).

From now on it will be assumed that $\alpha, \beta \in \mathbf{R}, \omega, R \in(0, \infty), \lambda \equiv \sqrt{\alpha^{2}+\beta^{2}} \in$ $\{0\} \cup(\omega, \infty)$ and

$$
S \equiv \frac{1}{R} T+\frac{2 \omega-1}{R} T_{1}+\frac{\lambda^{2}+\omega-\omega^{2}}{R}+i \alpha .
$$

The following technical lemma is needed.

LEMMA 2. Suppose that $\phi \in(0, \pi / 2]$ and

$$
a \leqslant \frac{\lambda^{2}}{2 R}-\frac{5 \lambda+4}{\sin \phi}-\frac{17\left|\omega^{2}-\omega\right|+4}{R \sin ^{2} \phi} .
$$

If $|\arg (z-a)| \in[\phi, \pi]$ then $z \notin \sigma(S)$ and

$$
\begin{gathered}
\left\|(S-z)^{-1}\right\| \leqslant \frac{4 R}{8 R+\lambda^{2} \sin \phi}, \quad\left\|(S-z)^{-1}\right\| \leqslant \frac{1}{2(\lambda+1)} \\
\left\|(S-z)^{-1}\right\| \leqslant \frac{4}{|z-a| \sin \phi}, \quad\left\|(S-z)^{-1}\right\| \leqslant \frac{8}{|z| \sin \phi} \\
\left\|T(S-z)^{-1}\right\| \leqslant \frac{2 R}{\sin \phi} .
\end{gathered}
$$


Proof. Define

$$
\begin{gathered}
r=\frac{\lambda^{2} \sin \phi}{2 R}+5 \lambda+4-|\alpha| \cos \phi+\frac{17\left|\omega^{2}-\omega\right|+4}{R \sin \phi}+\frac{\omega-\omega^{2}}{R} \sin \phi, \\
S_{1}=\frac{1}{R} T+\frac{\lambda^{2}+\omega-\omega^{2}}{R}+i \alpha-z .
\end{gathered}
$$

Lemma 1 implies

$$
\left\|T_{1} S_{1}^{-1}\right\| \leqslant\left\|S_{1}^{-1}\right\|^{1 / 2}\left\|T S_{1}^{-1}\right\|^{1 / 2} \leqslant\left(\frac{R}{r \sin \phi}\right)^{1 / 2} .
$$

So that

$$
\left\|\frac{2 \omega-1}{R} T_{1} S_{1}^{-1}\right\| \leqslant \frac{1}{2}, \quad(S-z)^{-1}=S_{1}^{-1}\left(1+\frac{2 \omega-1}{R} T_{1} S_{1}^{-1}\right)^{-1} .
$$

Bounds on $S_{1}$ as given by Lemma 1 imply Lemma 2 .

\subsection{Pressure.}

THEOREM 1. For every $v=\left(v_{1}, v_{2}, v_{3}\right) \in \mathcal{H}^{3}$ there exist a unique $u=\left(u_{1}, u_{2}, u_{3}\right)$ $\in \mathcal{H}^{3}$ with $u_{2} \in \mathcal{D}\left(T_{1}\right)$ and a unique $p \in \mathcal{D}\left(T_{0}\right)$ such that

$$
\begin{gathered}
v_{1}=u_{1}+i \alpha p, \quad v_{2}=u_{2}+\left(T_{0}-\omega\right) p, \\
v_{3}=u_{3}+i \beta p, \quad i \alpha u_{1}+i \beta u_{3}+\left(T_{1}-\omega\right) u_{2}=0 .
\end{gathered}
$$

Moreover, if $\lambda=0$ then $u_{1}=v_{1}, u_{2}=0, u_{3}=v_{3}$ and $p=-G(\omega) v_{2}$; and if $\lambda>\omega$ then

$$
\begin{aligned}
p & =F(\lambda-\omega) G(\lambda+\omega) v_{0}-(1 / \lambda) G(\lambda+\omega) v_{0}-(1 / \lambda) F(\lambda-\omega)\left(v_{0}-\lambda v_{2}\right), \\
u_{1} & =v_{1}-i \alpha p \\
u_{2} & =\lambda F(\lambda-\omega) G(\lambda+\omega) v_{0}-F(\lambda-\omega)\left(v_{0}-\lambda v_{2}\right), \\
u_{3} & =v_{3}-i \beta p
\end{aligned}
$$

where $v_{0}=\lambda v_{2}+i \alpha v_{1}+i \beta v_{3}$.

Proof. Do the obvious thing.

Let $p, u$ and $v$ be as in Theorem 1. Define $\hat{P}: \mathcal{H}^{3} \rightarrow \mathcal{H}^{3}$ and $\hat{\Pi}: \mathcal{H}^{3} \rightarrow \mathscr{H}$ by $\hat{P} v=u$ and $\hat{\Pi} v=p$. Let $\mathscr{N}$ be the range of $\hat{P}$, i.e. $\mathscr{K}=\left\{u \in \mathcal{H}^{3} \mid u=\right.$ $\left(u_{1}, u_{2}, u_{3}\right), u_{2} \in \mathscr{D}\left(T_{1}\right)$ and $\left.i \alpha u_{1}+i \beta u_{3}+\left(T_{1}-\omega\right) u_{2}=0\right\}$. Clearly, $\Re$ is a Hilbert space.

THEOREM 2. (a) If $\lambda=0$ then $\|\hat{P}\|=1$ and $\|\hat{\Pi}\|=1 / \omega$.

(b) If $\lambda>\omega$ then $\|\hat{P}\| \leqslant 5 \lambda^{2} /\left(\lambda^{2}-\omega^{2}\right)$ and $\|\hat{\Pi}\| \leqslant 4 \lambda /\left(\lambda^{2}-\omega^{2}\right)$.

Proof. Note that $\|F(z)\|=\|G(z)\|=1 / \operatorname{Re}(z)$ for $\operatorname{Re}(z)>0$. This fact and Theorem 1 imply (a). Let $p, u, v$ and $v_{0}$ be as in Theorem 1 and assume that $\lambda>\omega$. 
Define $a \equiv \sqrt{\left\|v_{1}\right\|^{2}+\left\|v_{3}\right\|^{2}}$, so that $\left\|v_{0}\right\| \leqslant \lambda\left(\left\|v_{2}\right\|+a\right)$ and $\left\|v_{0}-\lambda v_{2}\right\| \leqslant \lambda a$. Hence

$$
\begin{gathered}
\|p\| \leqslant \frac{\lambda}{\lambda^{2}-\omega^{2}}\left(\left\|v_{2}\right\|+a\right)+\frac{1}{\lambda+\omega}\left(\left\|v_{2}\right\|+a\right)+\frac{1}{\lambda-\omega} a \\
\leqslant \frac{\|v\|}{\lambda^{2}-\omega^{2}} \sqrt{(2 \lambda-\omega)^{2}+9 \lambda^{2}} \\
\left\|u_{1}\right\|^{2}+\left\|u_{3}\right\|^{2} \leqslant\left(\left\|v_{1}\right\|+\|\alpha p\|\right)^{2}+\left(\left\|v_{3}\right\|+\|\beta p\|\right)^{2} \leqslant(a+\lambda p)^{2} \\
\leqslant\left(\frac{\lambda^{2}\|v\|}{\lambda^{2}-\omega^{2}}\right)^{2}\left(\left(2-\frac{\omega}{\lambda}\right)^{2}+\left(4-\left(\frac{\omega}{\lambda}\right)^{2}\right)^{2}\right)
\end{gathered}
$$

and

$$
\left\|u_{2}\right\| \leqslant \frac{\lambda^{2}}{\lambda^{2}-\omega^{2}}\left\|v_{2}\right\|+\frac{2 \lambda^{2}+\omega \lambda}{\lambda^{2}-\omega^{2}} a \leqslant \frac{\lambda^{2}\|v\|}{\lambda^{2}-\omega^{2}} \sqrt{1+\left(2+\frac{\omega}{\lambda}\right)^{2}}
$$

These bounds give part (b).

2.3 The linear operator. In accordance with the perturbation equations define $\hat{A}$ : $\mathcal{H}^{3} \rightarrow \mathcal{H}^{3}$ by

$$
\begin{aligned}
& (\hat{A} u)_{1}=S u_{1}-i \alpha h_{1} u_{1}+h_{1} u_{2}, \\
& (\hat{A} u)_{2}=S u_{2}-i \alpha h_{1} u_{2} \\
& (\hat{A} u)_{3}=S u_{3}-i \alpha h_{1} u_{3}
\end{aligned}
$$

for $u=\left(u_{1}, u_{2}, u_{3}\right) \in \mathscr{D}(\hat{A})=\mathscr{D}(T) \times \mathscr{D}(T) \times \mathscr{D}(T)$. Define $A_{p}: \Re \rightarrow \Re$ by $\hat{A}_{p} u$ $=\hat{P} \hat{A} u$ for $u \in \mathscr{D}\left(\hat{A}_{p}\right)=\mathscr{D}(\hat{A}) \cap \mathfrak{R}$.

THEOREM 3. If $\alpha=0$ then $\sigma_{p}\left(\hat{A}_{p}\right)=\sigma_{p}(S)$ and $\sigma\left(\hat{A}_{p}\right)=\sigma(S)$.

THEOREM 4. If $\alpha \neq 0$ then

(a) $\sigma(S) \backslash \sigma_{p}(S) \subset \sigma\left(\hat{A}_{p}\right)$, and

(b) if $z \in \sigma\left(\hat{A}_{p}\right) \backslash \sigma(S)$ then $z$ is an isolated eigenvalue of $\hat{A}_{p},-2 /(1+2 \lambda)^{2}<$ $\operatorname{Im}(z) / \alpha<1$ and

$$
\begin{aligned}
& \operatorname{Re}(z)>\max \left\{\frac{\lambda^{2}+\gamma-\gamma^{2}}{R}-\frac{|\alpha|}{1+2 \lambda}\left(\frac{2 \lambda}{\lambda+\gamma+1}+\frac{1}{\lambda-\gamma+1}\right) \mid \gamma \in \mathbf{R},\right. \\
& |\gamma-1 / 2| \leqslant|\omega-1 / 2|\} \text {. }
\end{aligned}
$$

TheOREM 5. If $\omega \in(0,1)$ and $\operatorname{Re}(z) \leqslant 0$ then $z \in \sigma\left(\hat{A}_{p}\right)$ iff there exists $\phi \in$ $L^{2}(0, \infty)$ such that:

(1) $\phi^{\prime \prime \prime \prime}-2 \lambda^{2} \phi^{\prime \prime}+\lambda^{4} \phi=-\phi^{\prime \prime \prime}+\lambda^{2} \phi^{\prime}+R\left(i \alpha-i \alpha h_{1}-z\right)\left(\phi^{\prime \prime}-\lambda^{2} \phi\right)+$ $i \alpha R h_{1} \phi$,

(2) $\phi(0)=\phi^{\prime}(0)=0, \phi \nsucceq 0$,

(3) $\int_{0}^{\infty} e^{x}\left|\lambda \phi^{(j)}(x)+\phi^{(j+1)}(x)\right|^{2} d x<\infty$ for $j=0,1,2$. 
THEOREM 6. If $\phi \in(0, \pi / 2]$,

$$
a \leqslant \frac{\lambda^{2}}{2 R}-\frac{5 \lambda+4}{\sin \phi}-\frac{17\left|\omega-\omega^{2}\right|+4}{R \sin ^{2} \phi}
$$

and $|\arg (z-a)| \in[\phi, \pi]$ then $z \notin \sigma\left(\hat{A}_{p}\right)$ and

$$
\begin{aligned}
\left\|\left(\hat{A}_{p}-z\right)^{-1}\right\| & \leqslant \frac{16 R}{8 R+\lambda^{2} \sin \phi} \frac{\lambda+\omega}{|\lambda-\omega|}, \\
\left\|\left(\hat{A}_{p}-z\right)^{-1}\right\| & \leqslant \frac{16}{|z-a| \sin \phi} \frac{\lambda+\omega}{|\lambda-\omega|}, \\
\left\|\left(\hat{A}_{p}-z\right)^{-1}\right\| & \leqslant \frac{32}{|z| \sin \phi} \frac{\lambda+\omega}{|\lambda-\omega|}, \\
\left\|T^{(3)}\left(\hat{A}_{p}-z\right)^{-1}\right\| & \leqslant \frac{22 R}{\sin \phi} \frac{\lambda+\omega}{|\lambda-\omega|} .
\end{aligned}
$$

Theorems 3-6 will be proved in $\$ 2.4$ for the case $\lambda=0$; and in $\S 2.5$ for $\lambda>\omega$. The techniques used are similar to those in [11]. The essential observation is made in $\S 2.5$, Lemma 4. Much more could be said about the spectrum, see $[10,11]$.

Theorem 7. If $z \in \mathbf{C}, t>0, \phi \in(0, \pi / 2)$ and

$$
a \leqslant \frac{\lambda^{2}}{2 R}-\frac{5 \lambda+4}{\sin \phi}-\frac{17\left|\omega-\omega^{2}\right|+4}{R \sin ^{2} \phi},
$$

then

$$
\begin{aligned}
& \left\|e^{-\hat{A}_{p} t}\right\| \leqslant \frac{14}{\sin ^{2} \phi \cos \phi} \frac{\lambda+\omega}{|\lambda-\omega|} e^{-a t},
\end{aligned}
$$

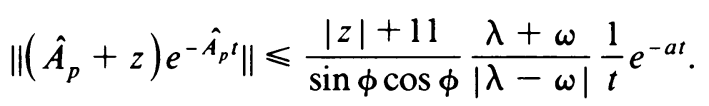

Proof. Let $a_{1}<a$ and $\phi_{1} \in(\phi, \pi / 2)$. For $x \in \mathbf{R}$ define $\gamma(x)=|x| \cos \phi_{1}-$ ix $\sin \phi_{1}+a_{1}$, so that

$$
\frac{1}{2 \pi} \int_{\mathbf{R}} e^{-\operatorname{Re}(t \gamma(x))}\left|\gamma^{\prime}(x)\right| d x=\frac{1}{\pi t \cos \phi_{1}} e^{-a_{1} t} .
$$

By Theorem 6

$$
\left\|\left(\gamma(x)-\hat{A}_{p}\right)^{-1}\right\| \leqslant \frac{\lambda+\omega}{|\lambda-\omega|} \frac{16}{\sin \phi} \frac{1}{\left(a-a_{1}\right) \sin \phi_{1}} .
$$

Hence [7,9]

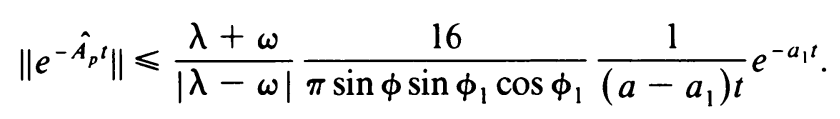

Now let $\left(a-a_{1}\right) t=1$ and $\phi_{1} \rightarrow \phi$.

By Theorem 6

$$
\left\|(z+\gamma(x))\left(\gamma(x)-\hat{A}_{p}\right)^{-1}\right\| \leqslant \frac{\lambda+\omega}{|\lambda-\omega|}\left(2|z|+\frac{32}{\sin \phi}\right)
$$


and hence

$$
\left\|\left(\hat{A}_{p}+z\right) e^{-\hat{A}_{p} t}\right\| \leqslant \frac{\lambda+\omega}{|\lambda-\omega|} \frac{|z|+11}{\sin \phi \cos \phi_{1}} \frac{1}{t} e^{-a_{1} t} .
$$

2.4 Case $\lambda=0$. Observe that in the case $\lambda=0, \Re=\left\{v=\left(v_{1}, 0, v_{3}\right) \mid v_{1}, v_{3} \in\right.$ $\mathcal{H}\},(\hat{A} u)_{1}=S u_{1},(\hat{A} u)_{2}=0$, and $(\hat{A} u)_{3}=S u_{3}$ for $u \in \mathscr{D}\left(\hat{A}_{p}\right)$. Thus, Theorem 3 holds. Lemma 2 implies Theorem 6. If $\omega \in(0,1)$ and $\operatorname{Re}(z) \leqslant 0$ then $z \notin \sigma\left(\hat{A}_{p}\right)$ by Lemma 1. It is easy to see that if $\operatorname{Re}(z) \leqslant 0$ then there is no $\phi \in L^{2}(0, \infty)$ for which conditions (1) $-(3)$ of Theorem 5 hold.

2.5 Case $\lambda>\omega$. Define $W: \mathscr{T} \rightarrow \mathcal{H}^{2}$ by $(W u)_{1}=\beta u_{1}-\alpha u_{3}$ and $(W u)_{2}=\lambda u_{2}-$ $i \alpha u_{1}-i \beta u_{3}$, where $u=\left(u_{1}, u_{2}, u_{3}\right) \in \mathcal{N}$.

LEMMA 3. $\|W\|=\lambda, W^{-1}$ exists and $\left\|W^{-1}\right\|=\sqrt{\lambda^{2}+\omega^{2}} /(\lambda(\lambda-\omega))$. If $w=$ $\left(w_{1}, w_{2}\right) \in \mathcal{H}^{2}$ then

$$
\begin{aligned}
& \left(W^{-1} w\right)_{1}=\frac{i \alpha w_{2}+\beta w_{1}}{\lambda^{2}}-\frac{i \alpha}{\lambda} F(\lambda-\omega) w_{2}, \\
& \left(W^{-1} w\right)_{2}=F(\lambda-\omega) w_{2}, \\
& \left(W^{-1} w\right)_{3}=\frac{i \beta w_{2}-\alpha w_{1}}{\lambda^{2}}-\frac{i \beta}{\lambda} F(\lambda-\omega) w_{2} .
\end{aligned}
$$

Furthermore, $W\left(\mathscr{D}\left(\hat{A}_{p}\right)\right)=\mathscr{D}(T) \times \mathscr{D}(T)$ and if $w \in \mathscr{D}(T) \times \mathscr{D}(T)$ then

$$
\left\|T^{(3)} W^{-1} w\right\| \leqslant \frac{\sqrt{\lambda^{2}+\omega^{2}}}{\lambda(\lambda-\omega)}\left\|T^{(2)} w\right\|+\sqrt{\frac{2}{\lambda-\omega}}\left\|T^{(2)} w\right\|^{3 / 4}\|w\|^{1 / 4} .
$$

Proof. Suppose that $u \in \mathcal{X}$ and $w=W u$. Then $\|w\|^{2}=\lambda^{2}\|u\|^{2}-2 \lambda \omega\left\|u_{2}\right\|^{2}$ and $\lambda^{2}\|u\|^{2}=\|w\|^{2}+2 \lambda \omega\left\|F(\lambda-\omega) w_{2}\right\|^{2}$. If $w \in \mathscr{D}(T) \times \mathscr{D}(T)$ then

$$
T^{(3)} W^{-1} w-W^{-1} T^{(2)} w=\left(-\frac{i \alpha}{\lambda} v, v,-\frac{i \beta}{\lambda} v\right)
$$

where $v=(T F(\lambda-\omega)-F(\lambda-\omega) T) w_{2}=-w_{2}^{\prime}(0) h_{\lambda-\omega}$. By Hölder's inequality $\left|w_{2}^{\prime}(0)\right| \leqslant \sqrt{2}\left\|T w_{2}\right\|^{3 / 4}\left\|w_{2}\right\|^{1 / 4}$ and therefore

$$
\left\|T^{(3)} W^{-1} w-W^{-1} T^{(2)} w\right\| \leqslant \sqrt{\frac{2}{\lambda-\omega}}\left\|T w_{2}\right\|^{3 / 4}\left\|w_{2}\right\|^{1 / 4} .
$$

Define $D: \mathcal{H}^{2} \rightarrow \mathcal{H}^{2}$ by

$$
\begin{aligned}
& (D w)_{1}=\left(S-i \alpha h_{1}\right) w_{1}+\beta h_{1} F(\lambda-\omega) w_{2}, \\
& (D w)_{2}=\left(S-i \alpha h_{1}+2 i \alpha \lambda G(\lambda+\omega) h_{1} F(\lambda-\omega)-i \alpha h_{1} F(\lambda-\omega)\right) w_{2}
\end{aligned}
$$

for $w=\left(w_{1}, w_{2}\right) \in \mathscr{D}(D)=\mathscr{D}(T) \times \mathscr{D}(T)$.

LEMMA 4. $\sigma_{p}\left(\hat{A}_{p}\right)=\sigma_{p}(D), \sigma\left(\hat{A}_{p}\right)=\sigma(D)$ and $\left(\hat{A}_{p}-z\right)^{-1}=W^{-1}(D-z)^{-1} W$ for $z \notin \sigma(D)$.

Proof. By Lemma 3 it is enough to show that $W \hat{A}_{p} u=D W u$ for all $u \in \mathscr{D}\left(\hat{A}_{p}\right)$. Let $u \in \mathscr{D}\left(\hat{A}_{p}\right)$ and $v=\hat{A} u$. Then

$$
\left(\hat{A}_{p} u\right)_{1}=v_{1}-i \alpha p=\left(S-i \alpha h_{1}\right) u_{1}+h_{1} u_{2}-i \alpha p,
$$




$$
\begin{aligned}
& \left(\hat{A}_{p} u\right)_{2}=v_{2}-\left(T_{0}-\omega\right) p=\left(S-i \alpha h_{1}\right) u_{2}-\left(T_{0}-\omega\right) p, \\
& \left(\hat{A}_{p} u\right)_{3}=v_{3}-i \beta p=\left(S-i \alpha h_{1}\right) u_{3}-i \beta p,
\end{aligned}
$$

for $p$ as in Theorem 1. $u_{2}=F(\lambda-\omega)(W u)_{2}$ by Lemma 3 and therefore

$$
\begin{aligned}
& \left(W \hat{A}_{p} u\right)_{1}=(D W u)_{1}, \\
& \left(W \hat{A}_{p} u\right)_{2}=\left(S-i \alpha h_{1}\right)(W u)_{2}-i \alpha h_{1} F(\lambda-\omega)(W u)_{2}-\lambda\left(\lambda-\omega+T_{0}\right) p .
\end{aligned}
$$

A simple computation of $\left(\lambda-\omega+T_{0}\right) p$ finishes the proof.

Define $B_{11}, B_{12}, B_{22} \in \mathscr{B}(\mathcal{H})$ by

$$
\begin{gathered}
B_{11}=-i \alpha h_{1}, \quad B_{12}=\beta h_{1} F(\lambda-\omega), \\
B_{22}=-i \alpha h_{1}+2 i \alpha \lambda G(\lambda+\omega) h_{1} F(\lambda-\omega)-i \alpha h_{1} F(\lambda-\omega) \\
=-i \alpha h_{1}+\frac{2 i \alpha \lambda}{1+2 \lambda} h_{1} G(\lambda+\omega+1)-\frac{i \alpha}{1+2 \lambda} F(\lambda-\omega+1) h_{1} .
\end{gathered}
$$

Denote

$$
B_{M}=\left(\begin{array}{ll}
B_{11} & B_{12} \\
0 & B_{22}
\end{array}\right)
$$

and $S^{(2)}=\left(\begin{array}{ll}S & 0 \\ 0 & S\end{array}\right), \mathscr{D}\left(S^{(2)}\right)=\mathscr{D}(T) \times \mathscr{D}(T)$. Therefore $D=S^{(2)}+B_{M}$.

LeMMA 5. (a) $\sigma(S) \backslash \sigma_{p}(S) \subset \sigma(D)$.

(b) If $z \in \sigma(D) \backslash \sigma(S)$ then $z$ is an isolated eigenvalue of $D$.

(c) $\sigma_{p}\left(S+B_{11}\right) \cup\left(\sigma_{p}\left(S+B_{22}\right) \backslash \sigma(S)\right) \subset \sigma_{p}(D) \subset \sigma_{p}\left(S+B_{11}\right) \cup \sigma_{p}\left(S+B_{22}\right)$.

Proof. $B_{M}$ is bounded and $S^{(2)}$-compact since $h_{1} \in L^{\infty}(0, \infty) \cap L^{2}(0, \infty)$ [9]. This implies (a) and (b) [9]. It is easy to verify part (c).

If $\alpha=0$ it is easy to see that $\sigma(D)=\sigma(S)$ and $\sigma_{p}(D)=\sigma_{p}(S)$ (also by Lemma 5). This proves Theorem 3. Lemmas 4 and 5 also imply Theorem 4 , except for the bounds on the eigenvalues.

Lemma 6. Suppose that $\gamma \in \mathbf{R},\left|\gamma-\frac{1}{2}\right| \leqslant\left|\omega-\frac{1}{2}\right|$ and $z \notin \sigma(S)$.

(a) If $z \in \sigma_{p}\left(S+B_{22}\right)$ then there exists $g \in \mathscr{D}(T), g \neq 0$, such that

$$
\begin{aligned}
z g=\left(\frac{1}{R} T\right. & +\frac{2 \gamma-1}{R} T_{1}+\frac{\lambda^{2}+\gamma-\gamma^{2}}{R}+i \alpha-i \alpha h_{1} \\
& \left.\quad+\frac{2 i \alpha \lambda}{1+2 \lambda} h_{1} G(\lambda+\gamma+1)-\frac{i \alpha}{1+2 \lambda} F(\lambda-\gamma+1) h_{1}\right) g .
\end{aligned}
$$

(b) If $z \in \sigma_{p}\left(S+B_{11}\right)$ then there exists $g \in \mathscr{D}(T), g \neq 0$, such that

$$
z g=\left(\frac{1}{R} T+\frac{2 \gamma-1}{R} T_{1}+\frac{\lambda^{2}+\gamma-\gamma^{2}}{R}+i \alpha-i \alpha h_{1}\right) g .
$$

(c) If $1+\gamma-\omega \geqslant 0$ then the converses of parts (a) and (b) hold.

Proof. Define $\xi=\sqrt{1 / 4+\lambda^{2}+R(i \alpha-z)}$ and note that $\operatorname{Re}(\xi)>\left|\omega-\frac{1}{2}\right|$. Observe also that if $\operatorname{Re}(s)>0, \operatorname{Re}(s+a)>0, f \in \mathcal{H}$ and $h_{a} f \in \mathcal{H}$ then $h_{a} F(s) f=$ $F(s+a) h_{a} f$ and $h_{a} G(s+a) f=G(s) h_{a} f$. 
Suppose that $z \in \sigma_{p}\left(S+B_{22}\right)$. Lemma 1 implies that there exists $f \in \mathscr{D}(T), f \neq 0$, such that

$$
f=-R F(\xi-\omega+1 / 2) G(\xi+\omega-1 / 2) h_{1} \psi
$$

where

$$
\psi=\left(-i \alpha+\frac{2 i \alpha \lambda}{1+2 \lambda} G(\lambda+\omega+1)-\frac{i \alpha}{1+2 \lambda} F(\lambda-\omega)\right) f .
$$

This implies that

$$
f=-R h_{\gamma-\omega} F(\xi-\gamma+1 / 2) G(\xi+\gamma-1 / 2) h_{1-\gamma+\omega} \psi .
$$

If $g \equiv h_{\omega-\gamma} f$ then

$$
\begin{array}{r}
g=-R F(\xi-\gamma+1 / 2) G(\xi+\gamma-1 / 2)\left(-i \alpha h_{1}+\frac{2 i \alpha \lambda}{1+2 \lambda} h_{1} G(\lambda+\gamma+1)\right. \\
\left.-\frac{i \alpha}{1+2 \lambda} F(\lambda-\gamma+1) h_{1}\right) g
\end{array}
$$

and this proves part (a). If $1+\gamma-\omega \geqslant 0$ then one can go backwards in a similar way. The same proof applies for part (b) and its converse.

The following lemma completes the proof of Theorem 4.

Lemma 7. (a) If $z \in \sigma_{p}\left(S+B_{22}\right) \backslash \sigma(S)$ then $\alpha \neq 0,-2 /(1+2 \lambda)^{2}<\operatorname{Im}(z) / \alpha<1$ and

$$
\begin{aligned}
& \operatorname{Re}(z)>\max \left\{\frac{\lambda^{2}+\gamma-\gamma^{2}}{R}-\frac{|\alpha|}{1+2 \lambda}\left(\frac{2 \lambda}{1+\lambda+\gamma}+\frac{1}{\lambda+1-\gamma}\right) \mid \gamma \in \mathbf{R},\right. \\
& |\gamma-1 / 2| \leqslant|\omega-1 / 2|\} \text {. }
\end{aligned}
$$

(b) If $z \in \sigma_{p}\left(S+B_{11}\right) \backslash \sigma(S)$ then $\alpha \neq 0,0<\operatorname{Im}(z) / \alpha<1$ and $\operatorname{Re}(z)>$ $\left(\lambda^{2}+1 / 4\right) / R$.

Proof. Suppose that $\gamma \in \mathbf{R},\left|\gamma-\frac{1}{2}\right| \leqslant\left|\omega-\frac{1}{2}\right|$ and $z \in \sigma_{p}\left(S+B_{22}\right) \backslash \sigma(S)$. By Lemma 6 there is $g \in \mathscr{D}(T), g \neq 0$, such that

$$
\begin{aligned}
(z-i \alpha- & \left.\frac{\lambda^{2}+\gamma-\gamma^{2}}{R}\right)\|g\|^{2}=\frac{1}{R}\left\|T_{1} g\right\|^{2}+\frac{2 \gamma-1}{R}\left(T_{1} g, g\right)-i \alpha\left(h_{1} g, g\right) \\
& +\frac{2 i \alpha \lambda}{1+2 \lambda}\left(h_{1} G(\lambda+\gamma+1) g, g\right)-\frac{i \alpha}{1+2 \lambda}\left(F(\lambda-\gamma+1) h_{1} g, g\right) .
\end{aligned}
$$

Therefore

$$
\left(\operatorname{Re}(z)-\frac{\lambda^{2}+\gamma-\gamma^{2}}{R}\right)\|g\|^{2}>-\frac{2|\alpha| \lambda}{1+2 \lambda} \frac{\|g\|^{2}}{\lambda+\gamma+1}-\frac{|\alpha|}{1+2 \lambda} \frac{\|g\|^{2}}{\lambda-\gamma+1} .
$$

By choosing $\gamma=\frac{1}{2}$ one obtains

$$
\begin{aligned}
\left(\frac{\operatorname{Im}(z)}{\alpha}-1\right)\|g\|^{2}= & -\left(h_{1} g, g\right)+\frac{2 \lambda}{1+2 \lambda} \operatorname{Re}\left(\left(h_{1} G\left(\lambda+\frac{3}{2}\right) g, g\right)\right) \\
& -\frac{1}{1+2 \lambda} \operatorname{Re}\left(\left(F\left(\lambda+\frac{1}{2}\right) h_{1} g, g\right)\right) .
\end{aligned}
$$


If $f=h_{1 / 2} g$ then

$$
\begin{aligned}
\frac{\operatorname{Im}(z)}{\alpha}\|g\|^{2}=\left(\left(1-h_{1}\right) g, g\right)+\frac{2 \lambda(\lambda+1)}{1+2 \lambda}\|F(\lambda+1) f\|^{2} & \\
& -\frac{1}{1+2 \lambda} \operatorname{Re}\left(\left(F(\lambda+1 / 2) h_{1} g, g\right)\right)>\frac{-2}{(1+2 \lambda)^{2}}\|g\|^{2}
\end{aligned}
$$

and

$$
\begin{aligned}
\left(\frac{\operatorname{Im}(z)}{\alpha}-1\right)\|g\|^{2} & =-\|f\|^{2}+\frac{2 \lambda(\lambda+1)}{1+2 \lambda}\|F(\lambda+1) f\|^{2}-\frac{\lambda}{1+2 \lambda}\|F(\lambda) f\|^{2} \\
& <\left(-1+\frac{2 \lambda}{1+2 \lambda} \frac{1}{\lambda+1}\right)\|f\|^{2}<0 .
\end{aligned}
$$

Lemma 8. Suppose that $\omega \leqslant \frac{3}{2}$ and $z \notin \sigma(S)$. Then $z \in \sigma_{p}\left(S+B_{22}\right)$ iff there exists $\phi \in L^{2}(0, \infty)$ such that:

(1) $\phi^{\prime \prime \prime \prime}-2 \lambda^{2} \phi^{\prime \prime}+\lambda^{4} \phi=-\phi^{\prime \prime \prime}+\lambda^{2} \phi^{\prime}+R\left(i \alpha-i \alpha h_{1}-z\right)\left(\phi^{\prime \prime}-\lambda^{2} \phi\right)+i \alpha R h_{1} \phi$.

(2) $\phi(0)=\phi^{\prime}(0)=0, \phi \neq 0$.

(3) $\int_{0}^{\infty} e^{x}\left|\lambda \phi^{(j)}(x)+\phi^{(j+1)}(x)\right|^{2} d x<\infty$ for $j=0,1,2$.

Proof. Suppose that $z \in \sigma_{p}\left(S+B_{22}\right)$. Let $g$ be as in Lemma 6(a) with $\gamma=\frac{1}{2}$. Define $\phi=F(\lambda) h_{1 / 2} g$. A straightforward computation shows that $\phi$ satisfies the above conditions. The converse statement follows from Lemma 6 in a similar way.

Thus, Theorem 5 is proved. Theorem 6 follows from Lemmas 3 and 4 and from the following observation.

LEMMA 9. Suppose that $\phi \in(0, \pi / 2]$,

$$
a \leqslant \frac{\lambda^{2}}{2 R}-\frac{5 \lambda+4}{\sin \phi}-\frac{17\left|\omega-\omega^{2}\right|+4}{R \sin ^{2} \phi}
$$

and $|\arg (z-a)| \in[\phi, \pi]$. Then $z \notin \sigma(D)$ and

$$
\begin{aligned}
& \left\|(D-z)^{-1}\right\| \leqslant \frac{16 R}{8 R+\lambda^{2} \sin \phi}, \quad\left\|(D-z)^{-1}\right\| \leqslant \frac{16}{|z-a| \sin \phi}, \\
& \left\|(D-z)^{-1}\right\| \leqslant \frac{32}{|z| \sin \phi}, \quad\left\|T^{(2)}(D-z)^{-1}\right\| \leqslant \frac{8 R}{\sin \phi} .
\end{aligned}
$$

Proof. Recall that $D=S^{(2)}+B_{M}$ and note that

$$
\begin{aligned}
\left\|B_{M}\right\|^{2} & \leqslant\left\|B_{11}\right\|^{2}+\left\|B_{22}\right\|^{2}+\left\|B_{12}\right\|^{2} \\
& \leqslant \alpha^{2}+\beta^{2}+\left(\lambda+\frac{\lambda}{2 \lambda+1} \frac{2 \lambda}{\lambda+\omega+1}+\frac{\lambda}{2 \lambda+1} \frac{1}{\lambda-\omega+1}\right)^{2} \\
& \leqslant \lambda^{2}+(\lambda+1)^{2} \leqslant\left(\frac{3}{2}(\lambda+1)\right)^{2}
\end{aligned}
$$

By Lemma $2\left\|B_{M}\left(S^{(2)}-z\right)^{-1}\right\| \leqslant\left\|B_{M}\right\|\left\|(S-z)^{-1}\right\| \leqslant \frac{3}{4}$ so that

$$
\left\|\left(1+B_{M}\left(S^{(2)}-z\right)^{-1}\right)^{-1}\right\| \leqslant 4 \text {. }
$$




\section{Solution.}

3.1 Preliminaries II. Fix $\alpha_{0}, \beta_{0} \in(0, \infty)$ and let $\Omega=\left[0,2 \pi / \alpha_{0}\right] \times[0, \infty) \times$ $\left[0,2 \pi / \beta_{0}\right]$. Denote by $\||\cdot|\|$ the usual norm on either $\mathcal{H}_{0}=L^{2}(\Omega)$ or $\mathcal{H}_{0}^{3} . L^{\infty}(\Omega)$ and $\left(L^{\infty}(\Omega)\right)^{3}$ norms will be denoted by \|\|$\cdot\|\|_{\infty}$, with convention that $\|u\|_{\infty}=$ $\max _{i}\left\{\left\|u_{i} \mid\right\|_{\infty}\right\}$ for $u=\left(u_{1}, u_{2}, u_{3}\right) \in\left(L^{\infty}(\Omega)\right)^{3}$.

For $n, m \in \mathbf{Z}$ and $x, z \in \mathbf{R}$ let $\phi_{n m}(x, z)=e^{i n \alpha_{0} x+i m \beta_{0} z}$. Recall that if $f_{n m} \in \mathscr{H}$ for $n, m \in \mathbf{Z}$ and $\Sigma_{n m}\left\|f_{n m}\right\|^{2}<\infty$ then $\sum_{n m} f_{n m}(y) \phi_{n m}(x, z)$ defines an $f \in \mathcal{K}_{0}$. Moreover, every $f \in \mathcal{H}_{0}$ can be uniquely expressed in that way. Thus, one can define

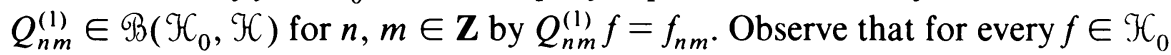

$$
\|f\|^{2}=\frac{(2 \pi)^{2}}{\alpha_{0} \beta_{0}} \sum_{n m}\left\|Q_{n m}^{(1)} f\right\|^{2} .
$$

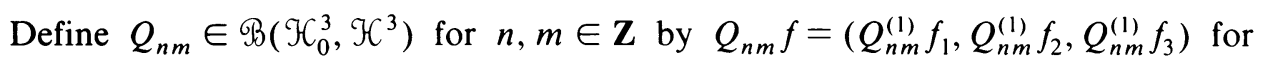
$f=\left(f_{1}, f_{2}, f_{3}\right) \in \mathcal{F}_{0}^{3}$.

Choose any $\omega>0$ such that $\omega<\alpha_{1} \equiv \min \left\{\alpha_{0}, \beta_{0}\right\} . R \in(0, \infty)$ is the Reynolds number. Fix any $n, m \in \mathbf{Z}$ and let $\alpha=n \alpha_{0}, \beta=m \beta_{0}$. Now, for these numbers $\alpha, \beta, \omega, R$ define

$$
\begin{aligned}
& \lambda_{n m}=\sqrt{\alpha^{2}+\beta^{2}} \in\{0\} \cup(\omega, \infty), \\
& \Re_{n m}=\Re \quad \text { as in } \S 2.2, \\
& P_{n m}=\hat{P} \quad \text { as in } \S 2.2, \\
& \Pi_{n m}=\hat{\Pi} \quad \text { as in } 2.2, \\
& A_{n m}=\hat{A} \quad \text { as in } \S 2.3, \\
& A_{p n m}=\hat{A_{p}} \quad \text { as in } \S 2.3, \\
& \Xi_{n m}(\phi)=\frac{\lambda_{n m}^{2}}{2 R}-\frac{5 \lambda_{n m}+4}{\sin \phi}-\frac{17\left|\omega-\omega^{2}\right|+4}{R \sin ^{2} \phi} \text { for } \phi \in\left(0, \frac{\pi}{2}\right] .
\end{aligned}
$$

3.2 Pressure. Define ("gradient") $C_{0}: \mathcal{H}_{0} \rightarrow \mathcal{H}_{0}^{3}$ by

(1) $f \in \mathscr{D}\left(C_{0}\right)$ iff $Q_{n m}^{(1)} f \in \mathscr{D}\left(T_{0}\right)$ for all $n, m \in \mathbf{Z}$ and

$$
\sum_{n m}\left\|T_{0} Q_{n m}^{(1)} f\right\|^{2}+\left(n^{2}+m^{2}\right)\left\|Q_{n m}^{(1)} f\right\|^{2}<\infty .
$$

(2) $Q_{n m} C_{0} f=\left(i n \alpha_{0} Q_{n m}^{(1)} f,\left(T_{0}-\omega\right) Q_{n m}^{(1)} f, i m \beta_{0} Q_{n m}^{(1)} f\right)$ for all $n, m \in \mathbf{Z}$ and all $f \in \mathscr{D}\left(C_{0}\right)$.

Define ("divergence") $C_{1}: \mathcal{H}_{0}^{3} \rightarrow \mathcal{H}_{0}$ by

(1) $f=\left(f_{1}, f_{2}, f_{3}\right) \in \mathscr{Q}\left(C_{1}\right)$ iff $Q_{n m}^{(1)} f_{2} \in \mathscr{D}\left(T_{1}\right)$ for all $n, m \in \mathbf{Z}$ and $\Sigma_{n m}\left\|i n \alpha_{0} Q_{n m}^{(1)} f_{1}+\left(T_{1}-\omega\right) Q_{n m}^{(1)} f_{2}+i m \beta_{0} Q_{n m}^{(1)} f_{3}\right\|^{2}<\infty$.

(2) $Q_{n m}^{(1)} C_{1} f=\operatorname{in} \alpha_{0} Q_{n m}^{(1)} f_{1}+\left(T_{1}-\omega\right) Q_{n m}^{(1)} f_{2}+i m \beta_{0} Q_{n m}^{(1)} f_{3}$ for all $f \in \mathscr{D}\left(C_{1}\right)$ and all $n, m \in \mathbf{Z}$.

The basic working space will be $X=\left\{f \in \mathscr{D}\left(C_{1}\right) \mid C_{1} f=0\right\}$, equipped with the $\mathcal{H}_{0}^{3}$ norm. Clearly, if $f \in \mathcal{H}_{0}^{3}$ then $f \in X$ iff $Q_{n m} f \in \mathcal{N}_{n m}$ for all $n, m \in \mathbf{Z}$.

THEOREM 8. For every $v \in \mathcal{H}_{0}^{3}$ there exists a unique $u \in X$ and a unique $p \in \mathscr{Q}\left(C_{0}\right)$ such that $v=u+C_{0} p$. Moreover, $Q_{n m} u=P_{n m} Q_{n m} v$ and $Q_{n m}^{(1)} p=\Pi_{n m} Q_{n m} v$ for all 
$n, m \in \mathbf{Z}$. Furthermore,

$$
\|u\| \leqslant \frac{5 \alpha_{1}^{2}}{\alpha_{1}^{2}-\omega^{2}} \mid\|v\| \| \quad \text { and } \quad\|p\| \mid \leqslant\|v\| \max \left\{\frac{1}{\omega}, \frac{4 \alpha_{1}}{\alpha_{1}^{2}-\omega^{2}}\right\} .
$$

Proof. See Theorems 1 and 2.

Let $u, v$ and $p$ be as in the theorem above. Define $P \in \mathscr{B}\left(\mathcal{H}_{0}^{3}\right)$ and $\Pi \in$ $\mathscr{B}\left(\mathcal{H}_{0}^{3}, \mathcal{H}_{0}\right)$ by $P v=u$ and $\Pi v=p$. Observe that $Q_{n m} P=P_{n m} Q_{n m}$ and $Q_{n m}^{(1)} \Pi=$ $\Pi_{n m} Q_{n m}$ for all $n, m \in \mathbf{Z}$.

3.3 The linearized operator. Define $A: \mathcal{H}_{0}^{3} \rightarrow \mathcal{H}_{0}^{3}$ by

(1) $u \in \mathscr{D}(A)$ iff $Q_{n m} u \in \mathscr{D}\left(T^{(3)}\right)$ for all $n, m \in \mathbf{Z}$ and $\Sigma_{n m}\left\|T^{(3)} Q_{n m} u\right\|^{2}+$ $\left(n^{2}+m^{2}\right)^{2}\left\|Q_{n m} u\right\|^{2}<\infty$.

(2) $Q_{n m} A u=A_{n m} Q_{n m} u$ for all $n, m \in \mathbf{Z}$ and all $u \in \mathscr{D}(A)$.

Define $A_{p}: X \rightarrow X$ by $\mathscr{Q}\left(A_{p}\right)=\mathscr{D}(A) \cap X$ and $A_{p} u=P A u$ for all $u \in \mathscr{D}\left(A_{p}\right)$. Observe that for all $u \in \mathscr{D}\left(A_{p}\right)$ and all $n, m \in \mathbf{Z}, Q_{n m} u \in \mathscr{D}\left(A_{p n m}\right)$ and $Q_{n m} A_{p} u=$ $A_{p n m} Q_{n m} u$.

THEOREM 9. (a) $\sigma_{p}\left(A_{p}\right)=\cup_{n m} \sigma_{p}\left(A_{p n m}\right), \sigma\left(A_{p}\right)=\cup_{n m} \sigma\left(A_{p n m}\right)$.

(b) If $u \in X$ and $z \notin \sigma\left(A_{p}\right)$ then $Q_{n m}\left(A_{p}-z\right)^{-1} u=\left(A_{p n m}-z\right)^{-1} Q_{n m} u$ for all $n, m \in \mathbf{Z}$.

(c) If $z \notin \sigma\left(A_{p}\right)$ then $A\left(A_{p}-z\right)^{-1} \in \mathscr{B}\left(X, \mathcal{H}_{0}^{3}\right)$.

(d) If $\phi \in(0, \pi / 2], a \leqslant \min _{n m}\left\{\Xi_{n m}(\phi)\right\}$ and $|\arg (z-a)| \in[\phi, \pi]$ then $z \notin \sigma\left(A_{p}\right)$,

$$
\begin{gathered}
\left\|\left(A_{p}-z\right)^{-1}\right\| \leqslant 2 \frac{\alpha_{1}+\omega}{\alpha_{1}-\omega}, \\
\left\|\left(A_{p}-z\right)^{-1}\right\| \leqslant \frac{16}{|z-a| \sin \phi} \frac{\alpha_{1}+\omega}{\alpha_{1}-\omega} .
\end{gathered}
$$

Proof. It is easy to see that $\sigma_{p}\left(A_{p}\right)=\cup_{n m} \sigma_{p}\left(A_{p n m}\right)$ and that $\cup_{n m} \sigma\left(A_{p n m}\right) \subset$ $\sigma\left(A_{p}\right)$. Fix $z \notin \cup_{n m} \sigma\left(A_{p n m}\right)$. By Theorem 6 there exists $c \in(0, \infty)$ such that $\left(1+n^{2}+m^{2}\right)\left\|\left(A_{p n m}-z\right)^{-1}\right\| \leqslant c$ and $\left\|T^{(3)}\left(A_{p n m}-z\right)^{-1}\right\| \leqslant c$ for all $n, m \in \mathbf{Z}$. This implies that $\sup _{n m}\left\{\left\|A_{n m}\left(A_{p n m}-z\right)^{-1}\right\|\right\}<\infty$. Thus, if $A_{1}: X \rightarrow X$ is defined by $Q_{n m} A_{1} v=\left(A_{p n m}-z\right)^{-1} Q_{n m} v, v \in X, n, m \in \mathbf{Z}$, then $A_{1} \in \mathscr{B}(X), A A_{1} \in \mathscr{B}\left(X, \mathcal{F}_{0}^{3}\right)$ and $A_{1} v \in \mathcal{Q}\left(A_{p}\right)$ for all $v \in X$. It is easy to see that $A_{1}\left(A_{p}-z\right) \subset\left(A_{p}-z\right) A_{1}=1$ and hence $A_{1}=\left(A_{p}-z\right)^{-1}$. This proves conclusions (a), (b) and (c). Part (d) follows from (b) and Theorem 6.

THEOREM 10. If $t>0, \gamma>0, a<\inf \operatorname{Re} \sigma\left(A_{p}\right), n, m \in \mathbf{Z}$ and $u \in X$ then $Q_{n m} e^{-A_{p} t} u=e^{-A_{p n m} t} Q_{n m} u$ and $Q_{n m}\left(A_{p}-a\right)^{-\gamma} u=\left(A_{p n m}-a\right)^{-\gamma} Q_{n m} u$.

Proof. Define $Q_{p n m} \in \mathscr{B}\left(X, \Re_{n m}\right)$ by $Q_{p n m} v=Q_{n m} v$ for $v \in X$. Then, for a suitable path in $\mathbf{C}[\mathbf{7 , 9 ]}$, one has

$$
\begin{aligned}
Q_{p n m} e^{-A_{p} t} & =\frac{1}{2 \pi i} Q_{p n m} \int\left(z-A_{p}\right)^{-1} e^{-t z} d z \\
& =\frac{1}{2 \pi i} \int\left(z-A_{p n m}\right)^{-1} e^{-t z} Q_{p n m} d z \\
& =e^{-A_{p n n} t} Q_{p n m} .
\end{aligned}
$$


Similarly [7],

$$
\begin{aligned}
Q_{p n m}\left(A_{p}-a\right)^{-\gamma} & =\frac{1}{\Gamma(\gamma)} Q_{p n m} \int_{0}^{\infty} s^{\gamma-1} e^{-\left(A_{p}-a\right) s} d s \\
& =\frac{1}{\Gamma(\gamma)} \int_{0}^{\infty} s^{\gamma-1} e^{-\left(A_{p n m}-a\right) s} Q_{p n m} d s \\
& =\left(A_{p n m}-a\right)^{-\gamma} Q_{p n m} .
\end{aligned}
$$

3.4 The nonlinear operator. A simliar version of the following theorem can be found in [2].

THEOREM 11. If $\gamma>\frac{3}{4}$ and $a<\inf \operatorname{Re} \sigma\left(A_{p}\right)$ then there exists $c \in(0, \infty)$ such that for all $v \in X,\left\|\left(A_{p}-a\right)^{-\gamma} v\right\|_{\infty} \leqslant c\|v\|$.

Proof. Define a norm on $\left(L^{\infty}(0, \infty)\right)^{3}$ by $\|u\|_{\infty}=\max _{i}\left\{\left\|u_{i}\right\|_{\infty}\right\}$ for $u=$ $\left(u_{1}, u_{2}, u_{3}\right) \in\left(L^{\infty}(0, \infty)\right)^{3}$. Note that if $f \in \mathscr{D}(T)$ then $\|f\|_{\infty} \leqslant \sqrt{2}\|T f\|^{1 / 4}\|f\|^{3 / 4}$. Fix $\phi \in(0, \pi / 2)$ and $s<\min _{n m}\left\{\Xi_{n m}(\phi)\right\}$. Then

$$
\begin{aligned}
\|u\|_{\infty} & \leqslant \sqrt{2}\left\|T^{(3)}\left(A_{p n m}-s\right)^{-1}\left(A_{p n m}-s\right) u\right\|^{1 / 4}\|u\|^{3 / 4} \\
& \leqslant \sqrt{2}\left(\frac{22 R}{\sin \phi} \frac{\alpha_{1}+\omega}{\alpha_{1}-\omega}\right)^{1 / 4}\left\|\left(A_{p n m}-s\right) u\right\|^{1 / 4}\|u\|^{3 / 4} \\
& \equiv c_{0}\left\|\left(A_{p n m}-s\right) u\right\|^{1 / 4}\|u\|^{3 / 4}
\end{aligned}
$$

for all $n, m \in \mathbf{Z}$ and all $u \in \mathscr{D}\left(A_{p n m}\right)$. Now, following [7], let $I_{n m}$ be the identity map from $\Re_{n m}$ into $\left(L^{\infty}(0, \infty)\right)^{3}$. Clearly, $I_{n m}$ is a closed linear operator and $I_{n m} e^{-A_{p n m} t} u$ is a continuous function of $t$ for $t>0$ and $u \in \Re_{n m}$. Hence for every $u \in \Re_{n m}$ the following holds:

$$
\begin{aligned}
\int_{0}^{\infty} t^{\gamma-1} & \left\|I_{n m} e^{-\left(A_{p n m}-s\right) t} u\right\|_{\infty} d t \\
& \leqslant c_{0} \int_{0}^{\infty} t^{\gamma-1}\left\|\left(A_{p n m}-s\right) e^{-\left(A_{p n m}-s\right) t} u\right\|^{1 / 4}\left\|e^{-\left(A_{p n m}-s\right) t} u\right\|^{3 / 4} d t \\
& \leqslant\|u\| c_{0} \int_{0}^{\infty} t^{\gamma-1}\left(\frac{|s|+11}{t \sin \phi \cos \phi}\right)^{1 / 4}\left(\frac{14}{\sin ^{2} \phi \cos \phi}\right)^{3 / 4} \frac{\alpha_{1}+\omega}{\alpha_{1}-\omega} e^{\left(s-\Xi_{n m}(\phi)\right) t} d t \\
& \equiv c_{1}\|u\|\left(\Xi_{n m}(\phi)-s\right)^{1 / 4-\gamma} .
\end{aligned}
$$

The last inequality is implied by Theorem 7 . Therefore

$$
\left\|I_{n m}\left(A_{p n m}-s\right)^{-\gamma} u\right\|_{\infty} \leqslant c_{2}\left(\Xi_{n m}(\phi)-s\right)^{1 / 4-\gamma}\|u\|
$$

for all $n, m \in \mathbf{Z}$ and all $u \in \mathcal{T}_{n m}$. Therefore, for every $v \in X$

$$
\begin{aligned}
\left\|\left(A_{p}-s\right)^{-\gamma} v\right\| \|_{\infty} & \leqslant \sum_{n m}\left\|I_{n m} Q_{n m}\left(A_{p}-s\right)^{-\gamma} v\right\|_{\infty}=\sum_{n m}\left\|I_{n m}\left(A_{p n m}-s\right)^{-\gamma} Q_{n m} v\right\|_{\infty} \\
& \leqslant c_{2} \sum_{n m}\left(\Xi_{n m}(\phi)-s\right)^{1 / 4-\gamma}\left\|Q_{n m} v\right\| \leqslant c_{3}\|v\|<\infty
\end{aligned}
$$


The following observation concludes the proof [7]:

$$
\left\|\left(A_{p}-a\right)^{-\gamma} v\right\|_{\infty}=\|\|\left(A_{p}-s\right)^{-\gamma}\left(A_{p}-s\right)^{\gamma}\left(A_{p}-a\right)^{-\gamma} v\left\|_{\infty} \leqslant c\right\|\|v\| .
$$

Define $B_{j}: \mathcal{H}_{0}^{3} \rightarrow \mathcal{H}_{0}^{3}$ for $j=1,2$ and 3 by

$$
\begin{aligned}
& \mathscr{D}\left(B_{1}\right)=\left\{u \in \mathcal{H}_{0}^{3} \mid \sum_{n m} n^{2}\left\|Q_{n m} u\right\|^{2}<\infty\right\}, \quad Q_{n m} B_{1} u=i n \alpha_{0} Q_{n m} u, \\
& \mathscr{D}\left(B_{2}\right)=\left\{u \in \mathcal{H}_{0}^{3} \mid Q_{n m} u \in \mathcal{D D}\left(T_{1}^{(3)}\right), \sum_{n m}\left\|T_{1}^{(3)} Q_{n m} u\right\|^{2}<\infty\right\},
\end{aligned}
$$

$$
Q_{n m} B_{2} u=T_{1}^{(3)} Q_{n m} u,
$$

$\mathcal{D}\left(B_{3}\right)=\left\{u \in \mathcal{H}_{0}^{3} \mid \sum_{n m} m^{2}\left\|Q_{n m} u\right\|^{2}<\infty\right\}, \quad Q_{n m} B_{3} u=i m \beta_{0} Q_{n m} u$.

The following theorem is similar to the preceding theorem and its proof is based on the same ideas $[2,7]$.

THEOREM 12. If $\gamma>\frac{1}{2}$ and $a<\inf \operatorname{Re} \sigma(A)$ then there is a $c \in(0, \infty)$ such that for all $v \in X$ and $j=1,2,3,\left\|B_{j}\left(A_{p}-a\right)^{-\gamma} v\right\|\|c\| v \|$.

Proof. Fix $\phi \in(0, \pi / 2)$ and $s<\min _{n m}\left\{\Xi_{n m}(\phi)\right\}$. Theorem 7 implies that for all $n, m \in \mathbf{Z}$

$$
\begin{aligned}
\left\|\left(A_{p n m}-s\right)^{-\gamma}\right\| & \leqslant \frac{1}{\Gamma(\gamma)} \int_{0}^{\infty} t^{\gamma-1}\left\|e^{-\left(A_{p n m}-s\right) t}\right\| d t \\
& \leqslant \frac{\alpha_{1}+\omega}{\alpha_{1}-\omega} \frac{14}{\sin ^{2} \phi \cos \phi}\left(\Xi_{n m}(\phi)-s\right)^{-\gamma}
\end{aligned}
$$

This proves the theorem for $j=1$ and 3 (and also for $\gamma=1 / 2$ ). Observe that for $n, m \in \mathbf{Z}$ and $t>0$ the function $t \rightarrow T_{1}^{(3)} e^{-A_{p m n} t}$ is continuous. Theorems 6 and 7 imply that

$$
\begin{aligned}
\left\|T_{1}^{(3)} e^{-\left(A_{p n m}-s\right) t}\right\| & \leqslant\left\|T^{(3)} e^{-\left(A_{p n m}-s\right) t}\right\|^{1 / 2}\left\|e^{-\left(A_{p n m}-s\right) t}\right\|^{1 / 2} \\
& \leqslant\left(\frac{22 R}{\sin \phi} \frac{\alpha_{1}+\omega}{\alpha_{1}-\omega}\right)^{1 / 2}\left\|\left(A_{p n m}-s\right) e^{-\left(A_{p n m}-s\right) t}\right\|^{1 / 2}\left\|e^{-\left(A_{p n m}-s\right) t}\right\|^{1 / 2} \\
& \leqslant c_{1} t^{-1 / 2} e^{-\left(\Xi_{n m}(\phi)-s\right) t}
\end{aligned}
$$

where $c_{1}$ does not depend on $n, m \in \mathbf{Z}$. Therefore, for all $n, m \in \mathbf{Z}$

$$
\left\|T_{1}^{(3)}\left(A_{p n m}-s\right)^{-\gamma}\right\| \leqslant \frac{c_{1} \Gamma(\gamma-1 / 2)}{\Gamma(\gamma)}\left(\Xi_{n m}(\phi)-s\right)^{1 / 2-\gamma}
$$

which proves the theorem for $j=2$.

Fix any $a<\inf \operatorname{Re} \sigma(A)$ and define [7] for $\gamma \geqslant 0$ a Banach space $X^{\gamma}$ with norm $\|\cdot\|_{\gamma}$ by $X^{\gamma}=\mathscr{D}\left(\left(A_{p}-a\right)^{\gamma}\right)$ and $\|x\|_{\gamma}=\left\|\left(A_{p}-a\right)^{\gamma} x\right\|$.

For $u=\left(u_{1}, u_{2}, u_{3}\right) \in X^{\gamma}, \gamma>\frac{3}{4}$, and $v \in X^{\delta}, \delta>1 / 2$, define $B(u, v) \in \mathcal{H}_{0}^{3}$ by

$$
B(u, v)=\left(h_{\omega} u_{1}\right) B_{1} v+\left(h_{\omega} u_{2}\right)\left(B_{2}-\omega\right) v+\left(h_{\omega} u_{3}\right) B_{3} v
$$


and let $B_{p}(u, v)=P B(u, v)$. Clearly, $B$ is a bilinear operator and for every $\gamma>\frac{3}{4}$ and $\delta>\frac{1}{2}$ there is $b$ such that $\|B(u, v)\| \leqslant b\|u\|_{\gamma}\|v\|_{\delta}$ for all $u \in X^{\gamma}$ and all $v \in X^{\delta}$.

3.5 Stability. For $t_{0} \in(0, \infty]$ define a set $\delta\left(t_{0}\right)$ by $x \in \mathcal{S}\left(t_{0}\right)$ iff:

(i) $x \in C\left(\left[0, t_{0}\right), X\right)$,

(ii) $x(t) \in \mathscr{D}(A)$ and $d x(t) / d t$ exists in $X$ for all $t \in\left(0, t_{0}\right)$,

(iii) for all $t \in\left(0, t_{0}\right)$ there exists $p(t) \in \mathcal{Q}\left(C_{0}\right)$ such that

$$
d x / d t+A x(t)+B(x(t), x(t))+C_{0} p(t)=0,
$$

and

(iv) $B_{p}(x(t), x(t))$ is a locally Hölder continuous function of $t \in\left(0, t_{0}\right)$ and $\int_{0}^{t}\|\| B_{p}(x(s), x(s)) \| d s<\infty$ for some $t>0$.

By Theorem 8, (iii) can be replaced by

(iii') $d x(t) / d t+A_{p} x(t)+B_{p}(x(t), x(t))=0$ for all $t \in\left(0, t_{0}\right)$.

Therefore, the results of D. Henry [7] are directly applicable.

Lemma 10 [7]. If $x \in \delta\left(t_{0}\right)$ then $x \in C\left(\left(0, t_{0}\right), X^{\delta}\right)$ for all $\delta \in[0,1]$ and for all $t \in\left[0, t_{0}\right)$

$$
x(t)=e^{-A_{p} t} x(0)-\int_{0}^{t} e^{-A_{p}(t-s)} B_{p}(x(s), x(s)) d s .
$$

Define $\delta^{\gamma}\left(t_{0}\right)$ for $\gamma \in\left(\frac{3}{4}, 1\right)$ and $t_{0} \in(0, \infty]$ by $\delta^{\gamma}\left(t_{0}\right)=\delta\left(t_{0}\right) \cap C\left(\left[0, t_{0}\right), X^{\gamma}\right)$.

THEOREM 13 [7]. If $\gamma \in\left(\frac{3}{4}, 1\right)$ and $x_{0} \in X^{\gamma}$ then there is $a t_{0}>0$ and a unique $x \in \mathcal{S}^{\gamma}\left(t_{0}\right)$ such that $x(0)=x_{0}$.

THEOREM 14 [7]. If $t_{0} \in(0, \infty), x \in \mathcal{S}\left(t_{0}\right)$ then either $\sup \left\{\|x(t)\|_{\gamma} \mid t_{0} / 2<t<t_{0}\right\}$ $=\infty$ for all $\gamma>\frac{3}{4}$ or there is $a t_{1}>t_{0}$ and $a y \in \delta\left(t_{1}\right)$ such that $y(t)=x(t)$ for all $0 \leqslant t<t_{0}$.

REMARK 1. In [7] it is assumed that $x(0) \in X^{\delta}$ for some $\delta \in\left(\frac{3}{4}, 1\right)$. This assumption can be easily removed by choosing an interior point for a new starting point.

THEOREM 15 [7]. If inf $\operatorname{Re} \sigma\left(A_{p}\right)>a>0$ and $\gamma \in\left(\frac{3}{4}, 1\right)$ then there exist $c_{1}, c_{2} \in$ $(0, \infty)$ such that for all $x_{0} \in X^{\gamma}$ with $\left\|x_{0}\right\|_{\gamma} \leqslant c_{1}$ there is a unique $x \in \delta^{\gamma}(\infty)$ with $x(0)=x_{0}$ and $\|x(t)\|_{\gamma} \leqslant c_{2}\left\|x_{0}\right\|_{\gamma} e^{-a t}$ for $t \geqslant 0$.

REMARK 2. Observe that under the above assumptions $\|x(t)\|\left\|_{\infty} \leqslant c_{3}\right\| x_{0} \|_{\gamma} e^{-a t}$ and $\|x(t)\| \leqslant c_{4}\left\|x_{0}\right\|_{\gamma} e^{-a t}$ for some $c_{3}$ and $c_{4}$ and for all $t \geqslant 0$. If, in addition, $x_{0} \in \mathscr{D}(A)$ then all $\left\|x_{0}\right\|_{\gamma}$ can be replaced with $\left\|A_{p} x_{0}\right\|$.

REMARK 3. If $\omega \in(0,1)$ then the sign $(+, 0,-)$ of inf $\operatorname{Re} \sigma\left(A_{p}\right)$ is determined by the classical Orr-Sommerfeld equation (Theorems 4, 5 and 9).

REMARK 4. If inf $\operatorname{Re} \sigma\left(A_{p}\right)<0$ then 0 is not stable [7].

\subsection{Smoothness.}

THEOREM 16 [7]. If $x \in \varsigma^{\gamma}\left(t_{0}\right)$ and $t_{1} \in\left(0, t_{0}\right)$ then there exist $c \in(0, \infty)$ and $\beta \in(0,1)$ such that $\|i x(t)-x(0)\|_{\infty} \leqslant c t^{\beta}$ for $t \in\left[0, t_{1}\right]$.

The following theorem is also expected [2]. 
TheOREM 17. If $x \in \mathcal{S}\left(t_{0}\right)$ then $x, A x \in C^{\infty}\left(\left(0, t_{0}\right), \mathcal{H}_{0}^{3}\right), x \in$ $C^{\infty}\left(\left(0, t_{0}\right),\left(L^{\infty}(\Omega)\right)^{3}\right)$ and $p \in C^{\infty}\left(\left(0, t_{0}\right), \mathcal{H}_{0}\right)$ where $p$ is as in the definition of $\mathcal{S}$ (§3.5).

Proof. Suppose that $x \in \mathcal{S}\left(t_{0}\right) \cap C^{n}\left(\left(0, t_{0}\right), X^{\gamma}\right)$ for some $n \geqslant 0$ and some $\gamma \in$ $\left(\frac{3}{4}, 1\right)$. By Lemma 10 this assumption is satisfied for $n=0$. Define $g(t)=$ $-B_{p}(x(t), x(t))$ for $0<t<t_{0}$.

Fix any $0<\tau<\tau_{1}<\tau_{2}<t_{0}$. There exists $c \in(0, \infty)$ such that for all $t, s \in\left[\tau_{1}, \tau_{2}\right]$

$$
\begin{gathered}
\|\| g^{(n)}(t)-g^{(n)}(s) \mid \| \leqslant c\left(|t-s|+\left\|x^{(n)}(s)-x^{(n)}(t)\right\|_{\gamma}\right), \\
x^{(n)}(t)=f(t)+\int_{\tau}^{t} e^{-A_{p}(t-u)} g^{(n)}(u) d u, \\
f(t)=\left(-A_{p}\right)^{n} e^{-A_{p}(t-\tau)} x(\tau)+\left(-A_{p}\right)^{n-1} e^{-A_{p}(t-\tau)} g(\tau) \\
+\cdots+e^{-A_{p}(t-\tau)} g^{(n-1)}(\tau) .
\end{gathered}
$$

There is a $d \in(0, \infty)$ such that

$$
\begin{gathered}
\left\|f^{\prime}(t)\right\|_{\gamma} \leqslant d \quad \text { for } t \in\left[\tau_{1}, \tau_{2}\right], \\
\left\|g^{(n)}(t)\right\| \leqslant d \quad \text { for } t \in\left[\tau, \tau_{2}\right], \\
\left\|e^{-A_{p} t}\right\|_{\gamma} \leqslant d t^{-\gamma} \quad \text { for } t \in\left(0, \tau_{2}\right], \\
\left\|\left(e^{-A_{p} h}-1\right) e^{-A_{p} t}\right\|_{\gamma} \leqslant d h t^{-\gamma-1} \quad \text { for } h, t \in\left(0, \tau_{2}\right] .
\end{gathered}
$$

Suppose that $\tau_{1}<t<t+h<\tau_{2}$. Then

$$
\begin{aligned}
x^{(n)}(t+h)-x^{(n)}(t)= & f(t+h)-f(t)+\int_{\tau}^{\tau_{1}}\left(e^{-A_{p} h}-1\right) e^{-A_{p}(t-u)} g^{(n)}(u) d u \\
& +\int_{\tau_{1}}^{\tau_{1}+h} e^{-A_{p}(t+h-u)} g^{(n)}(u) d u \\
& +\int_{\tau_{1}}^{t} e^{-A_{p}(t-u)}\left(g^{(n)}(u+h)-g^{(n)}(u)\right) d u
\end{aligned}
$$

so that

$$
\begin{aligned}
\left\|x^{(n)}(t+h)-x^{(n)}(t)\right\|_{\gamma} \leqslant & h\left(d+\frac{1}{\gamma} d^{2}\left(t-\tau_{1}\right)^{-\gamma}+d^{2}\left(t-\tau_{1}\right)^{-\gamma}\right) \\
& +d \int_{\tau_{1}}^{t}(t-u)^{-\gamma}\left\|g^{(n)}(u+h)-g^{(n)}(u)\right\| d u
\end{aligned}
$$

and therefore

$$
\begin{aligned}
& \left\|g^{(n)}(t+h)-g^{(n)}(t)\right\| \\
& \quad \leqslant c_{1} h\left(t-\tau_{1}\right)^{-\gamma}+c d \int_{\tau_{1}}^{t}(t-u)^{-\gamma}\left\|g^{(n)}(u+h)-g^{(n)}(u)\right\| d u
\end{aligned}
$$

for some constant $c_{1}$ (independent of $h$ and $t$ ). This is a modified Gronwall's inequality [7], therefore there is $c_{2} \in(0, \infty)$ such that for all $\tau_{1}<t<t+h<\tau_{2}$

$$
\left\|g^{(n)}(t+h)-g^{(n)}(t)\right\| \leqslant c_{2} h\left(t-\tau_{1}\right)^{-\gamma}
$$

and hence [7] the mapping $t \rightarrow \int_{\tau_{1}}^{t} e^{-A_{p}(t-s)} g^{(n)}(s) d s$ is in $C^{1}\left(\left(\tau_{1}, \tau_{2}\right), X^{\gamma}\right)$. Therefore $x^{(n)} \in C^{1}\left(\left(\tau_{1}, \tau_{2}\right), X^{\gamma}\right)$. 
By the induction principle $x \in C^{\infty}\left(\left(0, t_{0}\right), X^{\gamma}\right)$ and by Theorem $11 x \in$ $C^{\infty}\left(\left(0, t_{0}\right),\left(L^{\infty}(\Omega)\right)^{3}\right)$. It is clear that $x, x^{\prime}, g \in C^{\infty}\left(\left(0, t_{0}\right), X\right)$, hence $A_{p} x=g-x^{\prime}$ $\in C^{\infty}\left(\left(0, t_{0}\right), X\right)$. By Theorem $9 A x \in C^{\infty}\left(\left(0, t_{0}\right), \mathcal{H}_{0}^{3}\right)$. The observation

$$
p=\Pi\left(\left(A_{p}-A\right) x+B_{p}(x, x)-B(x, x)\right)
$$

completes the proof.

\section{REFERENCES}

1. P. G. Drazin and W. H. Reid, Hydrodynamic stability, Cambridge Univ. Press, New York, 1981.

2. H. Fujita and T. Kato, On the Navier-Stokes initial value problem, Arch. Rational Mech. Anal. 16 (1964), 269-315.

3. G. P. Galdi, Variational methods for stability of fluid motions in unbounded domains, Ricerche Mat. 27 (1978), 387-404.

4. G. P. Galdi and S. Rionero, On magnetohydrodynamic motions in unbounded domains: stability and uniqueness, Ann. Mat. Pura Appl. (4) 115 (1977), 119-154.

5. __ Local estimates and stability of viscous flows in an exterior domain, Arch. Rational Mech. Anal. (to appear).

6. __ A universal stability criterion in unbounded domains (to appear).

7. D. Henry, Geometric theory of semilinear parabolic equations, Lecture Notes in Math., vol. 840, Springer-Verlag, Berlin and New York, 1981.

8. D. D. Joseph, Stability of fluid motions. I, II, Springer-Verlag, Berlin and New York, 1976.

9. T. Kato, Perturbation theory for linear operators, 2nd ed., Springer-Verlag, Berlin and New York, 1980.

10. M. Miklavčič, Eigenvalues of the Orr-Sommerfeld equation in an unbounded domain, Arch. Rational Mech. Anal. (to appear).

11. M. Miklavčic and M. Williams, Stability of mean flows over an infinite flate plate, Arch. Rational Mech. Anal. 80 (1982), 57-69.

12. H. Schlichting, Boundary layer theory, McGraw-Hill, New York, 1955.

13. J. Serrin, On the stability of viscous fluid motions, Arch. Rational Mech. Anal. 3 (1959), 1-13.

InSTITUTE FOR MATHEMATICS AND ITS APPliCATIONS, University of Minnesota, Minneapolis, MiNNESOTA 55455

Current address: Majaronova 10, 61000 Ljubljana, Yugoslavia 